University of Massachusetts Amherst

From the SelectedWorks of Julian Tyson

1984

Extended Calibration of Flame AtomicAbsorption Instruments by a Flow Injection Peak Width Method

Julian Tyson 


\title{
Extended Calibration of Flame Atomic-absorption Instruments by a Flow Injection Peak Width Method
}

\author{
Julian F. Tyson \\ Department of Chemistry, University of Technology, Loughborough, Leicestershire, LE11 3TU, UK
}

\begin{abstract}
The principles of two existing methods of calibration based on flow injection methodology are outlined and the mathematical basis of a method based on peak width is presented. On the basis of the single well stirred tank model, it is shown that peak width at any concentration is proportional to logarithm of a function of concentration. The relationship is verified experimentally over the range 1-1000 p.p.m. of magnesium. The advantages and limitations of the method are discussed.
\end{abstract}

Keywords: Atomic-absorption calibration; flow injection analysis; peak width

The principles of two flow injection based procedures have already been described. ${ }^{1}$ The first of these procedures is based on the use of an exponential concentration gradient (with an accurately known concentration - time relationship) generated by the passage of a step concentration change through a well stirred mixing chamber (volume approximately $8 \mathrm{ml}$ ) located just in front of the nebuliser. If the instrument response is recorded as a function of time as the concentration gradient is introduced, then this record is, in fact, a calibration graph for the instrument as the time axis can be replaced by a concentration axis from the known relationship between concentration and time. Samples are introduced in the conventional manner and steady-state values used. Implementation of the method in practice will be discussed in detail elsewhere. ${ }^{2}$ The second method provides a means of carrying out the standard additions method of analysis and is based on injection of pure standards into the sample, used as the carrier stream. The method is based on controlling the dispersion of the standard within the sample carrier stream so that the interference effects act on the standards to the same extent as on the analyte. Details of the calculations required to select the required dispersion have already been given, ${ }^{3}$ and an example of the application of the method will be given elsewhere. ${ }^{4}$

One of the problems encountered in routine analytical situations is that the analyte concentrations of the sample solutions often do not fall within the rather limited range of concentrations (about one order of magnitude) that a typical flame atomic-absorption instrument can deal with at a given relative standard deviation. ${ }^{5}$ Approaches to dealing with samples of concentrations below this range will not be discussed further here. For samples whose concentrations are above this range, a variety of methods have been used. The instrument sensitivity can be reduced by either changing the absorbing path length, usually by rotation of the burner, or selection of a less sensitive line from the light source or reducing the nebulisation efficiency by adjustment of uptake rate or spray production mechanics. Alternatively, the sample may be diluted. This can be done without using pipettes and calibrated flasks by a suitable flow injection manifold. The most elegant method is that of "zone sampling"; after injection of the discrete sample volume, a slice of the dispersed sample zone is injected by a second injection system into the carrier stream flowing to the spectrometer. By controlling the timing of the second injection a variety of dilutions can be achieved. ${ }^{6}$

A new method of dealing with sample solutions that are too concentrated for the normal calibration range is proposed here, based on measurement of the width of peaks produced on flow injection introduction.

\section{Basis of Peak Width Method}

Assume the peaks to be composed of exponential rise and fall curves as would be obtained by passage of a volume $V_{i}$ of concentration $C_{\mathrm{m}}$ through a single well stirred mixing chamber of volume $V$ at flow-rate $u$. As the leading edge of the sample plug enters the mixing chamber the change in concentration as a function of time is given by

$$
\frac{\mathrm{d} c}{\mathrm{~d} t}=\frac{C_{\mathrm{m}} u}{V}-\frac{C u}{V}
$$

Separating the variables and integrating gives

$$
\ln \left(C_{\mathrm{m}}-C\right)=\frac{-u t}{V}+k
$$

where $k$ is a constant of integration. Putting in the initial conditions $t=0, C=0$ gives $k=\ln C_{m}$. Therefore,

$$
t=\frac{V}{u} \ln \left(\frac{C_{\mathrm{m}}}{C_{\mathrm{m}}-C}\right)
$$

$\{$ this can be further rearranged to give the more familiar form $\left.C=C_{\mathrm{m}}[1-\exp (-u t / V)]\right\}$.

The peak concentration, $C_{\mathrm{p}}$, is achieved as the trailing edge of the sample plug just enters the mixing chamber at a time given by

$$
t_{\mathrm{p}}=\frac{V_{i}}{u}
$$

Thereafter the rate of change of concentration with time (measured from the peak maximum) is given by

$$
\frac{\mathrm{d} c}{\mathrm{~d} t}=-C_{\mathrm{p}} \frac{u}{V}
$$

Integrating as above gives

$$
t=\frac{V}{u} \ln \left(\frac{C_{\mathrm{p}}}{C}\right)
$$

[which can similarly be rearranged to give the more familiar form $\left.C=C_{\mathrm{p}} \exp (-u t / V)\right]$.

Hence the peak width $t^{\prime}$ at any concentration $C^{\prime}$ is given by

$$
t^{\prime}=\frac{V_{i}}{u}-\frac{V}{u} \ln \left(\frac{C_{\mathrm{m}}}{C_{\mathrm{m}}-C^{\prime}}\right)+\frac{V}{u} \ln \left(\frac{C_{\mathrm{p}}}{C^{\prime}}\right)
$$

This is shown in Fig. 1.

Rearranging and substituting the dispersion, $D=C_{\mathrm{m}} / C_{\mathrm{p}}$, gives

$$
t^{\prime}=\frac{V}{u} \ln \left(\frac{C_{\mathrm{m}}}{C^{\prime}}-1\right)-\frac{V}{u} \ln (D-1)
$$




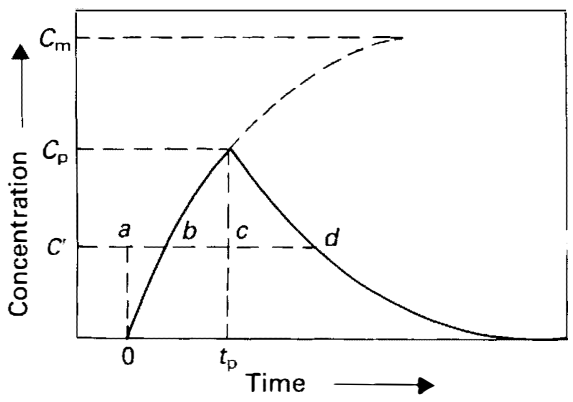

Fig. 1. Exponential rise and fall curves for peak produced by passage of sharp concentration boundaries through a well stirred mixing chamber. Peak width, $t^{\prime}$, is given by $t^{\prime}=b d=a c-a b+c d$. $C_{\mathrm{m}}$ is the concentration of the injected solution, $C_{\mathrm{p}}$ is the concentration at the peak maximum and $C^{\prime}$ is the concentration level chosen for measurement

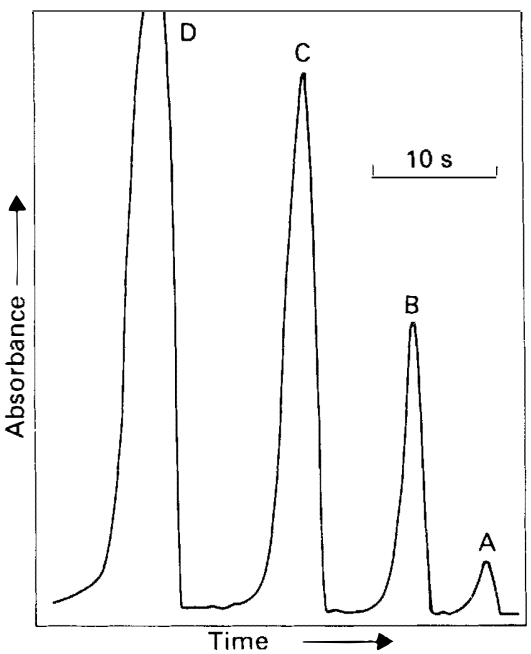

Fig. 2. Chart recordings of typical peaks produced from injection of approximately $100 \mu \mathrm{l}$ of $\mathrm{A}, 1 ; \mathrm{B}, 10 ; \mathrm{C}, 100 ;$ and $\mathrm{D}, 1000 \mu \mathrm{g} \mathrm{ml}-1$ of magnesium

Thus a plot of $t^{\prime}$ versus $\ln \left[\left(C_{\mathrm{m}} / C^{\prime}\right)-1\right]$ for different values of $C_{\mathrm{m}}$ should be a straight line of slope $V / u$ and intercept $(V / u) \ln (D-1)$.

\section{Experimental}

\section{Apparatus}

A Perkin-Elmer 290B atomic-absorption spectrometer was used with magnesium as the test element. Approximately 100 $\mu \mathrm{l}$ of standard solution were injected via a Rheodyne Model 5020 rotary injection valve into a carrier stream in $0.58 \mathrm{~mm}$ i.d. PTFE tubing propelled by an Ismatec Model Mini-S 840 peristaltic pump at a flow-rate of $6.9 \mathrm{ml} \mathrm{min}^{-1}$. The minimum length of tubing possible was used between injection valve and nebuliser.

The dispersion produced is thus dominated by the nebuliser performance characteristics producing a good approximation to the single well stirred tank model. The hypothetical tank volume is approximately $80 \mu \mathrm{l}$.

The spectrometer response was monitored with a Philips Model PM8251 chart recorder.

\section{Standard Solutions}

Solutions of concentration 1,10 and 100 p.p.m. were prepared by serial dilution of a stock 1000 p.p.m. solution (BDH Chemicals). All four were used for the peak-width calibration. Solutions covering the range $0-10$ p.p.m. were used for a conventional calibration.

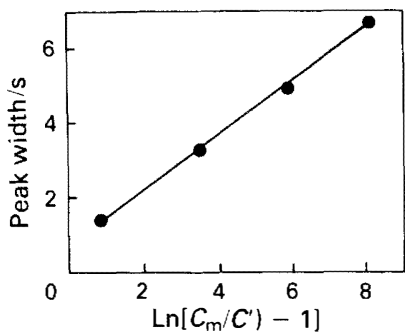

Fig. 3. Graph of peak width, $t^{\prime}$, against $\ln \left[\left(C_{\mathrm{m}} / C^{\prime}\right)-1\right]$

Table 1. Data for peak width calibration method

\begin{tabular}{lcccc} 
Peak width/mm & $\begin{array}{c}\text { Mean peak } \\
\text { width/s }\end{array}$ & $C_{\mathrm{m}}$, p.p.m. & $\frac{C_{\mathrm{m}}}{C^{\prime}}-1$ & $\ln \left(\frac{C_{\mathrm{m}}}{C^{\prime}}-1\right)$ \\
$7.0,7.0,7.0$ & 1.4 & 1.00 & 2.33 & 0.85 \\
$16.0,17.0,16.5$ & 3.30 & 10.0 & 32.3 & 3.48 \\
$24.5,24.5,24.5$ & 4.90 & 100 & 332 & 5.81 \\
$33.5,34.0,33.5$ & 6.86 & 1000 & 3332 & 8.11 \\
\hline
\end{tabular}

\section{Procedure}

After optimising the instrument response for magnesium and selecting a suitable flow-rate, a conventional calibration was obtained by continuously pumping each standard in turn and noting the steady-state absorbance. The peak-width calibration data were obtained by injecting, in triplicate, approximately $100 \mu \mathrm{l}$ of each of the standards into a distilled water carrier stream and recording the resulting peak at the recorder's fastest speed of $300 \mathrm{~mm} \mathrm{~min}^{-1}$. A suitable absorbance value corresponding to $C^{\prime}$ in equation (4) was selected and the peak widths were measured to the nearest 0.5 $\mathrm{mm}$.

\section{Results}

Typical peak shapes for each of four concentrations are shown in Fig. 2. The absorbance scale for the instrument used is arbitrary, but the cut-off at full-scale deflection of the chart recorder for peak d corresponds approximately to 0.5 absorbance unit. The absorbance level selected for peak-width measurements corresponded to a concentration of 0.30 p.p.m. The peak-width calibration data are given in Table 1 and a plot of peak width, $t^{\prime}$, against $\ln \left(C_{\mathrm{m}} / C^{\prime}-1\right)$ is shown in Fig. 3. A least-squares fit of the best straight line gave a slope of 0.745 , intercept 0.716 and correlation coefficient 0.999 .

\section{Discussion}

The basis of the peak-width method of calibration is that the peaks are exponential in shape. The linear relationship obtained here supports previously reported peak shapes ${ }^{3}$ arising from the injection of solutions close to the nebuliser, confirming the validity of the single well stirred tank model already proposed for this experimental arrangement. As the peak width can only be measured to the nearest $0.5 \mathrm{~mm}$ from the chart recording, this gives an uncertainty of about $\pm 5 \%$ relative over the whole concentration range. This could be improved by increasing the slope of the calibration plot by changing the experimental conditions either to increase $V$ or to decrease $u$, or both. Processing the spectrometer response with a microcomputer would also lead to an improvement by decreasing the uncertainty associated with measuring the time of the peak width. The method will probably never be as accurate as the conventional calibration procedure owing to the uncertainties in the measured quantities, but would prove useful in providing guidelines for dilution factors for subsequent accurate analysis and of course, is applicable in 
situations where the best accuracy is not required. As a strict adherence to a linear relationship is not necessary for a calibration function, some approximations in the method could be tolerated. For example, $\ln C_{\mathrm{m}}$ could be used instead of $\ln \left(C_{\mathrm{m}} / C^{\prime}-1\right)$ in the calibration function. A plot of $t^{\prime}$ versus $\ln C_{\mathrm{m}}$ for the data listed in Table 1 gives an almost identical correlation coefficient. The peak width should be measured at an absorbance value within the linear part of the absorbance concentration relationship in order that the absorbance analogue of equation (4) is valid. However, this restriction could probably be relaxed and this, together with the use of $\ln C_{\mathrm{m}}$ in the plot, would avoid the need to determine the concentration corresponding to the absorbances at which the peak width was measured. Finally, strict adherence to exponential peak shapes and the conditions necessary to produce them is probably also not necessary as whatever shapes the peaks are, it is likely that the function relating peak width and the logarithm of the concentration will increase monotonically.

\section{Conclusion}

The concentration gradients produced by allowing concentration boundaries to disperse during laminar flow in narrowbore tubing under precisely controlled conditions have been shown to provide the basis for several different calibration procedures. The combination of flow injection sample introduction techniques with microcomputer data storage and manipulation would appear to offer some interesting possibilities with direct advantages to the practising analyst, not just in the field of atomic-absorption spectrometry but also in other areas of routine instrumental analysis.

\section{References}

1. Tyson, J. F., Appleton, J. M. H., and Idris, A. B., Analyst, $1983,108,153$.

2. Tyson, J. F., and Appleton, J. M. H., Talanta, in the press.

3. Tyson, J. F., Appleton, J. M. H., and Idris, A. B., Anal. Chim. Acta, 1983, 145, 159

4. Tyson, J. F., and Idris, A. B., Analyst, 1984, 109, 23.

5. van Dalen, H. P. J., and de Galan, L., Analyst, 1981, 106, 695

6. Reis, B. F., Jacintho, A. O., Morlatti, J., Krug, F. J., Zagatto E. A. G., Bergamin Fo. H., and Pessenda, L. C. R., Anal. Chim. Acta, 1981, 123, 221. 